\title{
Dual-Region Location Management for Mobile Ad Hoc Networks
}

\author{
Yinan Li, Ing-Ray Chen \\ Department of Computer Science \\ Virginia Tech \\ Email: \{yinan926, irchen\}@vt.edu
}

\author{
Ding-Chau Wang \\ Department of Information Management \\ Southern Taiwan University \\ Email: dcwang@mail.stut.edu.tw
}

\begin{abstract}
We propose and analyze a novel location management scheme for mobile ad hoc networks (MANETs) called Dualregion Mobility Management (DrMoM). The basic design concept of DrMoM is to use local regions to complement existing location services in MANETs that assign home regions to mobile nodes and have mobile nodes in the home region of a mobile node serve as location servers for that node. DrMoM is based on the design notion of integrated mobility and service management for network cost minimization. Specifically, unlike existing location services that define the home region size statically at design time for all mobile users, DrMoM dynamically determines the optimal home region size and local region size per mobile user based on mobility and service characteristics of individual mobile nodes to minimize the overall network cost incurred by location management and data packet delivery. We develop a performance model to derive the optimal values of these two key design parameters under which the overall network cost incurred by DrMoM is minimized. Through a comparative performance study, we show that DrMoM outperforms a well-known scheme called SLURP based on static home regions as well as a regionbased location management scheme called RUDLS which claims to outperform contemporary region-based location management schemes.
\end{abstract}

\section{INTRODUCTION}

A Mobile Ad hoc Network (MANET) is a self-organizing and self-configuring network, in which mobile nodes form and maintain a dynamic network topology without a fixed infrastructure. While location management research is well developed for wireless mesh networks $[1,2,3,4]$, cellular networks [5,6,7], and Mobile IP networks [8], scalable location management for MANETs is still an open issue [9]. A recent study [10] reveals that hierarchical region-based location management $[9,11,12,13,14]$ is the most promising location management scheme for achieving scalability and efficiency.

A prevalent region-based location service in MANETs is hashing-based with which each mobile node is assigned a home region through hashing $[15,16,17,18,19]$. The nodes in the home region serve as location servers for that mobile node. A mobile node sends location updates to its location servers when it moves. To locate a destination node, a source node sends a location query to the destination node's location servers. Although a hashing-based location service is highly scalable, it has a major drawback: a source node has to contact the location servers of the destination node regardless of how close it is away from the destination node. If the two nodes are close to each other, contacting the location servers which may be far away geographically incurs unnecessary overhead. One way to solve this problem is to have a mobile node periodically exchange up-to-date location information with neighboring nodes in a local region [20,21]. If some node in the local region of the source node knows the location of the destination node, the source node can locate the destination node utilizing only local location information from the neighboring nodes, without having to query the destination node's home region. It is also possible that the source node is within the local region of the destination node and therefore knows where the destination node is located using only local location information it keeps.

In this paper, we propose and analyze a scalable, efficient location management scheme for location-based routing in MANETs called Dual-region Mobility Management (DrMoM) based on the idea of employing local regions to complement existing home region based location service schemes in MANETs that assign home regions to mobile nodes and have mobile nodes in the home region of a mobile node serve as location servers for that node. Relative to existing work utilizing home region based location service $[15,16,17,18,19]$ and local region based location service $[9,11,12,13,14,20,21]$, our contribution is to dynamically determine the optimal home region size and local region size for $e$ ach mobile node based on the mobile node's runtime mobility and service characteristics to minimize network cost.

DrMoM is based on the design notion of integrated mobility and service management for network cost minimization $[2,8]$. Specifically, unlike existing location services that define the home region size statically at design time, DrMoM dynamically determines the optimal home region size and local region size (defined by their respective radii denoted by $R_{h}$ and $R_{l}$ ), which together minimize the overall network cost incurred by location management and data packet delivery. We develop a performance model for deriving the optimal values of the two key design parameters $R_{h}$ and $R_{l}$ and for calculating the overall network cost incurred by DrMoM, given system parameters characterizing the mobility and service characteristics of mobile nodes. To demonstrate the benefit of our dual-region location management scheme, we compare location-based routing based on DrMoM against a wellknown scheme called SLURP [16] based on static home regions as well as a region-based location management scheme 
called RUDLS [14] which claims to outperform contemporary region-based location management schemes. We show that DrMoM outperforms both SLURP and RUDLS in terms of the overall network cost incurred.

The paper is organized as follows. Section II describes our scalable design for DrMoM. Section III presents a performance model for analytically evaluating the performance of DrMoM. Section IV performs a comprehensive performance evaluation, focusing on the effect of various parameters on the performance of DrMoM, as well as a comparative performance analysis of DrMoM against SLURP and RUDLS. Finally Section V summarizes the paper, discusses the applicability, and outlines future research areas.

\section{Dual-Region Mobility Management For LOCATION-BASED ROUTING}

We assume that mobile nodes are capable of tracking their locations, moving direction, and moving speed via a GPS module. We also assume that the density of mobile nodes is sufficiently high, so there is always at least one location server in each node's home region.

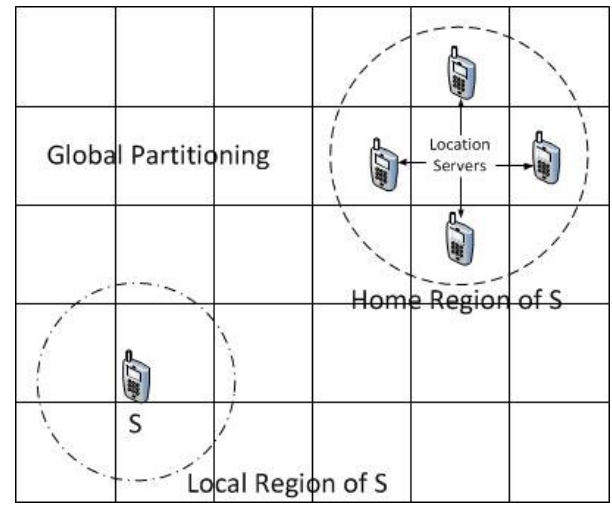

Fig. 1. Global partitioning of the MANET coverage area into rectangular regions.

In DrMoM, the coverage area of a MANET is statically partitioned into equally sized rectangular regions, as shown in Fig. 1. This global partitioning of the MANET coverage area is used as the basis for home region assignment. Specifically, each mobile node is permanently assigned a home region, whose center co-locates with the center of one of the rectangular regions, as illustrated by Fig. 1. The assignment is calculated by hashing the unique ID of the mobile node (e.g., its IP or MAC address) to the ID of one of the rectangular regions. We assume that every mobile node has knowledge about the global partitioning as well as the hash function such that it is able to locate the center of the home region of any node. All mobile nodes within the home region of a mobile node serve as location servers for that node. DrMoM varies the home region size dynamically based on the mobile node's runtime mobility and service characteristics. The home region size can be expanded as needed to ensure that at least one node exists to serve as the location server.
Besides the home region, each mobile node is also associated with a local region, and it exchanges location information with neighbors in the local region. Unlike the home region, which does not move, the local region moves with the mobile node. Local region location updates follow a threshold-based approach. Specifically, a mobile node broadcasts a location update to its neighbors within its local region, when the distance between its current location and the location where the last update was triggered exceeds a threshold. Each mobile node maintains a variable that records the location where the last local region location update was performed. Given a chosen threshold, the frequency of local region location updates depends on the node mobility rate [22]. In this paper, the threshold is set to be equal to the wireless transmission range $(r)$ such that the difference between the location of a mobile node kept by neighbors in its local region and its actual location is never larger than the wireless transmission range. Note that because the local region of a node is not restricted to its one-hop transmission range, a neighbor could potentially be multiple hops away. The home region keeps location summary information of the node, i.e., the coordinate of the center and radius of the node's local region. Whenever the local region moves due to movement of the node, the location servers in the home region are updated with the location summary information. To locate the local region of a destination node, the source node sends a location query to the destination node's location servers.

The coordinates of the center of a home region is statically determined, whereas the radius is dynamically determined on a per-node basis, depending on the node's mobility and service characteristics. The home region size, determined by its radius denoted by $R_{h}$, is a key factor balancing the tradeoff between the overhead for location queries/updates and the robustness of the location service. Specifically, a larger home region covers more location servers on average and consequently increases the chance of a successful location query. However, a larger home region also leads to larger overhead for location queries and updates. Because $R_{h}$ is dynamic, the size of the home region is dynamic and not necessarily restricted by the size of the rectangular region. The local region size, determined by its radius denoted by $R_{l}$, is also a key parameter. Increasing the local region size increases the chance that a destination node is located using local location information, without querying the location servers. However, as the local region size increases, the cost of location inquiry packet delivery increases because of more hops to travel. The local region size also impacts on the rate of location updates to the home region, which is equal to the rate of local region boundary crossing.

Each mobile node maintains two location tables: the local region location table $L T_{l}$ that stores location information of nodes for which it is within their local regions, and the home region location table $L T_{h}$ that stores location information of nodes for which it serves as a location server. $L T_{l}$ is updated whenever the mobile node receives a local region location update, whereas $L T_{h}$ is updated whenever it receives a home region location update. An entry in $L T_{l}$ keeps the correspond- 
ing node's "exact" location obtained from the most recent local region location update from that node. An entry in $L T_{h}$ stores the coordinates of the center and radius of the corresponding node's local region obtained from the most recent home region location update from that node. A timestamp is associated with each entry in the tables to indicate its freshness and is copied into the header of data packets when the entry (for the destination) is used by the source node for data packet delivery. Expired table entries are deleted periodically to make room for new entries.

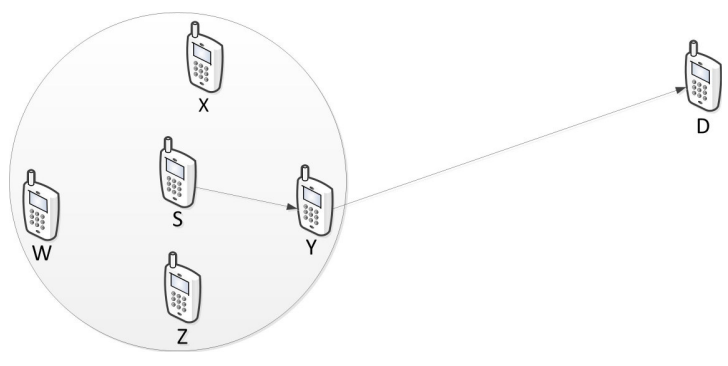

Fig. 2. Greedy geographical packet forwarding.

DrMoM uses geographical routing to route data packets and control messages such as messages for location updates and queries, as illustrated in Fig. 2. For each hop, DrMoM selects the node from the one-hop neighbors of the current node that is closest to the destination (i.e., the node that makes the most progress towards the destination) as the next forwarding node. For example, in Fig. 2, node $Y$ is selected by the source $S$ as the next forwarding node because it is closest to the destination $D$ among the neighbors of $S$. By selecting the next forwarding node this way, DrMoM guarantees that progress is made towards the destination for each hop, finally leading to the destination.

\section{PERformance Model}

In this section, we present a performance model for calculating the parameterized overall communication cost incurred by DrMoM as a function of $R_{l}$ and $R_{h}$. We define the total communication cost incurred by DrMoM for location management and data packet delivery by the total number of wireless transmissions per time unit. It is worth emasizing that because the total communication cost is a per time unit metric, a small amount of communication cost savings can be significant over time. Also note that we use the total communication cost as the performance metric here because the focus of this paper is on integrated mobility and service management for minimizing the total communication cost. We believe that minimizing the total communication cost will have a significant positive impact on other performance metrics, such as end-to-end packet delay and packet delivery ratio. This is because reducing the total number of wireless transmissions per time unit results in less wireless transmission conflicts and better wireless channel utilization, and consequently a larger probability of successful packet deliveries and a shorter average packet delay. It also has the benefit of maximizing the life time of a MANET since minimizing the total number of wireless transmissions per time unit means minimized battery consumption. Table I lists the notations used for model parameters.

TABLE I

THE NOTATIONS USED IN PERFORMANCE ANALYSIS.

\begin{tabular}{|ll|}
\hline Notation & Meaning \\
\hline$n$ & total number of mobile nodes in the MANET \\
$r$ & wireless transmission range \\
$R_{l}$ & radius of a local region \\
$R_{h}$ & radius of a home region \\
$b(R)$ & broadcast cost in a region with radius $R$ \\
$v$ & moving speed (m/s) of a mobile node \\
$\sigma$ & crossing rate of local region boundaries of a mobile node \\
$\bar{d}$ & average distance between a node and its home region \\
$\alpha$ & average number of hops between a node and its home region \\
$\gamma$ & node density (average number of nodes per unit area) \\
$\lambda_{l}$ & rate of local region location updates \\
$\lambda_{h}$ & rate of home region location updates \\
$\mu$ & rate of home region maintenance \\
$\phi$ & data packet rate \\
$\zeta$ & session rate \\
\hline
\end{tabular}

We make the following assumptions when building the performance model:

- We use the modified random way point mobility model to simulate the movement of mobile nodes. Specifically, each node picks a random point and it moves towards that point with velocity $v$ randomly chosen in the range $\left[v_{\min }, v_{\max }\right] . v_{\min }$ is positive to avoid the problem of speed decay as time progresses. Once the point is reached, the node chooses a new random point and moves towards the new point without a pause.

- We assume that the hash function used for home region assignment maps any mobile node uniformly to any rectangular region with equal probability.

According to [16], as a mobile node moves, the rate $\sigma$ at which it crosses local region boundaries can be calculated as:

$$
\sigma=\frac{v \pi}{4 R_{l}}
$$

Because a home region location update is triggered every time a local region boundary crossing occurs, the rate of home region location updates $\lambda_{h}$ is equal to $\sigma$. Local region location updates are triggered whenever the distance between the current location and the location where the last update happened exceeds the threshold $\tau$, which is equal to the wireless transmission range. Thus, the rate of local region location updates $\lambda_{l}$ of a mobile node depends on the wireless transmission range $r$ and the moving speed $v$ of the node, computed as follows:

$$
\lambda_{l}=\frac{v}{r}
$$

The broadcast cost $b(R)$ in a region with radius $R$ is defined as the number of wireless transmissions to cover the entire region, and can be approximated as follows [16]:

$$
b(R)=1+\frac{\pi R^{2}}{\pi r^{2}}=1+\frac{R^{2}}{r^{2}}
$$


Assume that the geographic area of the MANET is an $m \times m$ square. The average distance $\bar{d}$ between any mobile node and its home region in the $m \times m$ square area can be estimated as [23]:

$$
\bar{d}=\frac{2 m}{3}
$$

Therefore, the average number of hops $\alpha$ between any mobile node and its home region in the $m \times m$ square area can be approximated as follows:

$$
\alpha=\frac{\bar{d}}{r}
$$

\section{A. Location Update Cost $C_{u}$}

The location update cost $C_{u}$ consists of two parts: $C_{u}^{l}$, the cost for local region location updates, and $C_{u}^{h}$, the cost for home region location updates. A local region location update from a mobile node $S$ requires broadcasting the location update message among the neighbors in $S$ 's local region, thus incurring a broadcast cost of $b\left(R_{l}\right)$. A home region location update requires sending the location update message to $S$ 's home region that incurs a cost of $\alpha$, followed by a broadcast of the message within $S$ 's home region that adds a broadcast cost of $b\left(R_{h}\right)$. Therefore, $C_{u}^{l}$ and $C_{u}^{h}$ are calculated respectively as follows:

$$
\begin{aligned}
& C_{u}^{l}=b\left(R_{l}\right) \\
& C_{u}^{h}=\alpha+b\left(R_{h}\right)
\end{aligned}
$$

\section{B. Location Query Cost $C_{q}$}

The location query cost $C_{q}$ consists of the cost for a local region location query and optionally the cost for a home region location query which happens only when the local region location query fails. Let $C_{q}^{l}$ and $C_{q}^{h}$ denote the cost for a location region location query and the cost for a home region location query, respectively. Let $p_{q}^{h}$ denote the probability that the home region location query is needed to locate the target mobile node $D$, i.e., $p_{q}^{h}$ is the probability that the local region location query fails. $C_{q}$ is calculated as follows:

$$
C_{q}=C_{q}^{l}+p_{q}^{h} \cdot C_{q}^{h}
$$

A local region location query requires broadcasting the location query message among the neighbors in the local region of the source mobile node $S$, and collecting replies from these neighbors. Therefore, the cost for a local region location query consists of the broadcast cost $b\left(R_{l}\right)$ in the source mobile's local region and the cost for the neighbors who keep valid location information of $D$ to send the relies back to $S$. The number of neighbors in $S$ 's local region who keep the location information of $D$ can be estimated based on the node density. Specifically, a neighbor in $S$ 's local region keeps the updated location information of $D$ when it is also within $D$ 's local region or home region, the probability of which is $\frac{\pi R_{l}^{2}+\pi R_{h}^{2}}{m^{2}}$, assuming that the $n$ mobile nodes are uniformly distributed in the network. Therefore, the number of neighbors who keep the location information of $D$ can be estimated as follows:

$$
\frac{\pi R_{l}^{2}+\pi R_{h}^{2}}{m^{2}} \cdot \pi R_{l}^{2} \cdot \gamma
$$

Given the estimated number of neighbors in $S$ 's local region who have the location information of $D, C_{q}^{l}$ can thus be estimated as:

$$
C_{q}^{l}=b\left(R_{L}\right)+\frac{\pi R_{l}^{2}+\pi R_{h}^{2}}{m^{2}} \cdot \pi R_{l}^{2} \cdot \gamma
$$

A home region location query requires sending the location query message to $D$ 's home region, followed by forwarding the location reply back to $S$. Therefore, the cost for the home region location query $C_{q}^{h}$ consists of the costs for sending the location query message and location reply message, calculated as follows:

$$
C_{q}^{h}=2 \alpha
$$

$S$ needs to initiate a home region location query only if the local region location query fails when none of the mobile nodes in $S$ 's local region could find a valid entry for $D$ in their $L T_{l}$ and $L T_{h}$. A mobile node in $S$ 's local region could not find a valid entry for $D$ if it's not in $D$ 's local region and home region, the probability of which is $1-\frac{\pi R_{l}^{2}}{m^{2}}-\frac{\pi R_{h}^{2}}{m^{2}} \cdot p_{q}^{h}$ is the probability that all nodes in $S$ 's local region are not in $D$ 's local region or home region, which is computed as follows :

$$
p_{q}^{h}=\left(1-\frac{\pi R_{l}^{2}}{m^{2}}-\frac{\pi R_{h}^{2}}{m^{2}}\right)^{\pi R_{l}^{2} \cdot \gamma}
$$

\section{Data Packet Delivery Cost $C_{d}$}

Suppose the source node $S$ has a data packet $m$ to send to the destination node $D . S$ needs to locate $D$ first by looking up the location information of $D$ in its $L T_{l}$ and $L T_{h}$. Depending on the result of this table lookup, there could be three cases as follows:

- Case 1: A valid entry for $D$ exists in $L T_{l}$.

- Case 2: A valid entry for $D$ exists in $L T_{h}$.

- Case 3: No valid entry for $D$ can be found because the entry has expired or no entry for $D$ exists. In this case, $S$ initiates a location query before sending any data packets to $D$. Upon receiving the location reply, $S$ updates its location tables and follows geographic routing to do data packet delivery.

Let $C_{d}^{1}$ and $C_{d}^{2}$ denote the cost for data packet delivery for the first two cases.. Also let $p_{1}$ and $p_{2}$ denote the probability that a valid entry is found in $L T_{l}$ and the probability that a valid entry is found in $L T_{h}$, respectively, then $C_{d}$ is calculated as:

$$
C_{d}=p_{1} \cdot C_{d}^{1}+p_{2} \cdot C_{d}^{2}+\left(1-p_{1}-p_{2}\right) \cdot C_{q}
$$

Data delivery in the first case only involves mobile nodes in $S$ 's local region that make progress moving data packets towards $D$, and the distance from $S$ to $D$ is bound by the diameter of the region $2 R_{l}$. Therefore, we can estimate an upper bound of $C_{d}^{1}$ as follows:

$$
C_{d}^{1}=\frac{2 R_{l}}{r}
$$

Data delivery in the second case consists of two stages: the first stage routes the data packet from $S$ to the first mobile node (say $X$ ) on the route that is within $D$ 's local region, and 
the second stage is equivalent to data delivery in the first case, except that the source mobile node is $X$. Therefore, we can estimate $C_{d}^{2}$ as follows:

$$
C_{d}^{2}=\alpha+C_{d}^{1}
$$

The source mobile node $S$ can find a valid entry in either $L T_{l}$ or $L T_{h}$ only if $S$ is within the local region or home region of $D$. The probability $p_{1}\left(p_{2}\right)$ that $S$ is within the local region (home region) of $D$ can be calculated as follows, assuming that the $n$ mobile nodes are evenly distributed in the MANET:

$$
\begin{aligned}
& p_{1}=\frac{\pi R_{l}^{2}}{m^{2}} \\
& p_{2}=\frac{\pi R_{h}^{2}}{m^{2}}
\end{aligned}
$$

\section{Home Region Maintenance Cost $C_{m}$}

As discussed above, DrMoM handles the case that a mobile node $B$ enters into the home region of another node $A$ and becomes a location server for $A$ by requiring each node in $A$ 's home region to periodically broadcast an announcement message to its neighbors within its wireless transmission range. This incurs a home region maintenance cost $C_{m}$, consisting of the cost incurred for one wireless transmission by each node in the home region. Therefore, the calculation of $C_{m}$ is shown as follows:

$$
C_{m}=\pi R_{h}^{2} \cdot \gamma
$$

\section{E. Total Communication Cost $C$}

The total communication cost consists of the data packet delivery cost $\left(C_{d}\right)$, the location update cost $\left(C_{u}\right)$, the location query cost $\left(C_{q}\right.$, which is contained in the data delivery cost), and the home region maintenance cost $\left(C_{m}\right)$, multiplied by their rates respectively. $C$ is calculated as follows:

$$
C=\phi \cdot C_{d}+\lambda_{l} \cdot C_{u}^{l}+\lambda_{h} \cdot C_{u}^{h}+\mu \cdot C_{m}
$$

\section{Performance Evaluation}

We consider a scenario that $n$ mobile nodes are evenly distributed in an area of dimensions $2000 \mathrm{~m}$ by $2000 \mathrm{~m}$. $n$ varies from 100 to 800 with an increment of 100 , so that the density of nodes is a function of $n$. The wireless transmission range is $200 \mathrm{~m}$. We model the data stream between a source and a destination using a constant-bit-rate (CBR) stream at a rate of $\phi=50$ packets $/ \mathrm{s}$. The moving speed of mobile nodes $(v)$ varies between $1 \mathrm{~m} / \mathrm{s}$ to $20 \mathrm{~m} / \mathrm{s}$.

\section{A. Performance Characteristics of DrMoM}

We first evaluate the effect of $R_{l}\left(R_{h}\right)$ on the performance of DrMoM by varying the value of $R_{l}\left(R_{h}\right)$ but keeping $R_{h}$ $\left(R_{l}\right)$ fixed. Fig. 3 and Fig. 4 show the total communication cost as a function of $R_{l}$ and $R_{h}$, respectively, for a scenario where $n=100$ and $v=2 \mathrm{~m} / \mathrm{s}$. As can be seen in the figures, both $R_{l}$ and $R_{h}$ are key parameters and have a significant effect of the total communication cost incurred by DrMoM. More importantly, there exists optimal $R_{l}\left(R_{h}\right)$ that minimizes the total communication cost incurred by DrMoM. Increasing $R_{l}$ of a mobile node (and thus the area of the local region) increases the chance that the node is located utilizing only

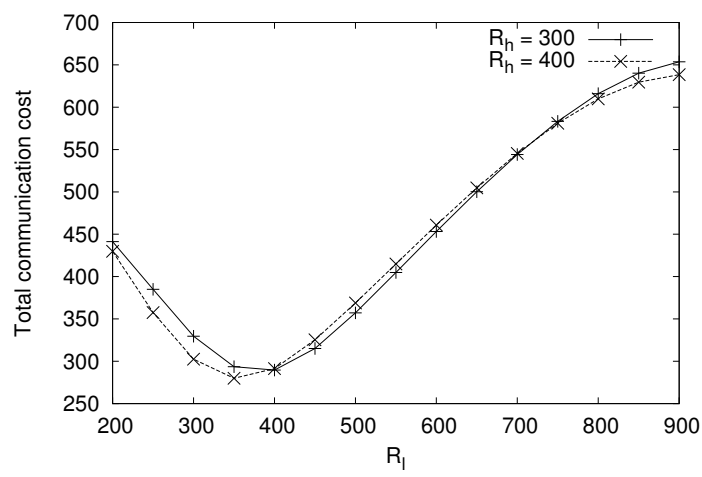

Fig. 3. Total communication cost vs. $R_{l}$ in DrMoM.

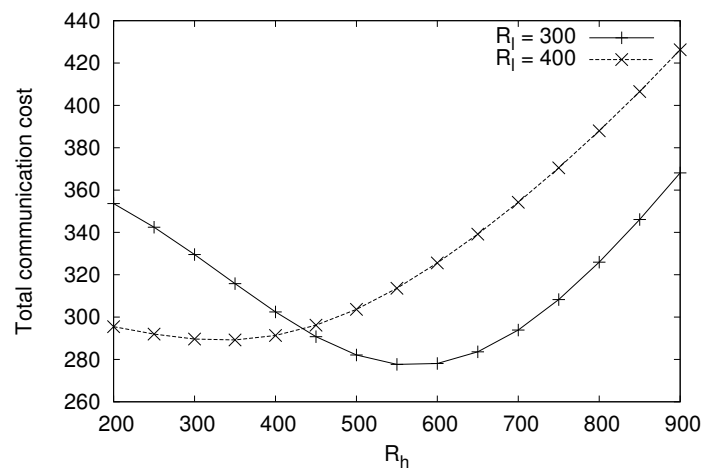

Fig. 4. Total communication cost vs. $R_{h}$ in DrMoM.

local location information, but it also increases the location update cost as well as the data delivery cost because a data packet tends to travel a longer distance in the local region after it reaches the first node within the local region. The same reasoning applies to $R_{h}$.

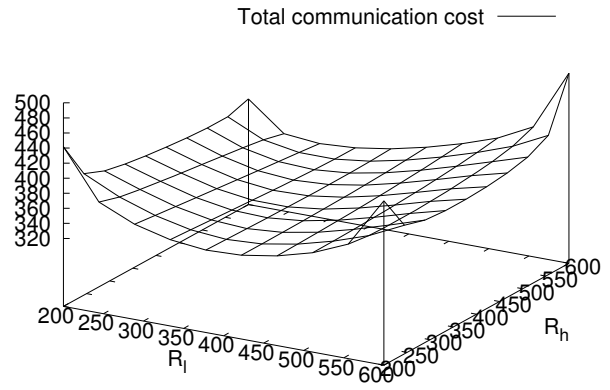

Fig. 5. Total communication cost vs. $R_{l}$ and $R_{h}$ in DrMoM.

Fig. 5 further shows the total communication cost incurred by DrMoM as a function of both $R_{l}$ and $R_{h}$. The figure depicts the effect of the interaction between $R_{l}$ and $R_{h}$ on the total communication cost incurred by DrMoM, and it justifies that there exists an optimal combination of $R_{l}$ and $R_{h}$ that 
minimizes the total communication cost incurred by DrMoM. It can also be seen in the figure that the total communication cost increases sharply when $R_{l}$ and/or $R_{h}$ are too large or too small.

\section{B. Performance Comparison}

In this section, we compare DrMoM with a well known location-based routing protocol called SLURP [16] based on static home regions as well as a region-based location management scheme called RUDLS [14] which claims to outperform contemporary region-based location management schemes. in terms of the overall network cost incurred.

SLURP handles location management using a scalable location service based on statically partitioned and assigned home regions. When a mobile node moves, it updates its location with the location servers in its home region by sending location update messages. To locate a destination mobile node $D$, the node's home region is queried to locate the home region in which $D$ currently resides. Geographical routing is used to forward a data packet sent to $D$ towards the center of the local region of $D$. When the data packet arrives at the first node within the local region, Dynamic Source Routing (DSR) is employed to deliver the data packet to $D$ within the region. SLURP defines the region size statically when the coverage area of a MANET is partitioned into grids, each of which corresponds to a region. This can be interpreted as having statically and equally sized home regions and local regions in DrMoM. Therefore, SLURP can be viewed as a special case of DrMoM.

RUDLS [14] on the other hand is a region-based location management scheme consisting of level 1 and level 2 location servers. Each level 1 location server keeps track of the locations of mobile users in its region each covering 9 grids. When a mobile user moves from one grid to another grid within the same region, only the location database of the level 1 location server is updated. On the other hand, each level 2 location server covers a number of level 1 location servers (e.g., $9 \times 9$ grids). When a mobile user moves from one level 1 region to another level 1 region, the location database of the level 2 location server is updated. Finally, when a mobile user moves from one level 2 region to another level 2 region, all level 2 location servers are updated with the user's new location, which is an expensive location update operation. A location query always goes bottom-up, i.e., it will go from the local level 1 location server and if necessary to the local level 2 location server, and if necessary, to a remote level 2 location server.

To make a fair comparison of DrMoM against SLURP and RUDLS, we use the same parameter values as reported in [16] and evaluate their performance under identical settings.

Fig. 6 compares the total communication cost incurred per time unit by DrMoM vs. SLURP and RUDLS as a function of the packet arrival rate $\phi$ in the range of 10 to 50 packets $/ s$ for the scenario in which $n=100$ and $v=2 \mathrm{~m} / \mathrm{s}$. It shows that the overall communication cost per time unit per user increases linearly with the packet arrival rate. Fig. 7 compares the total
Total Communication Cost

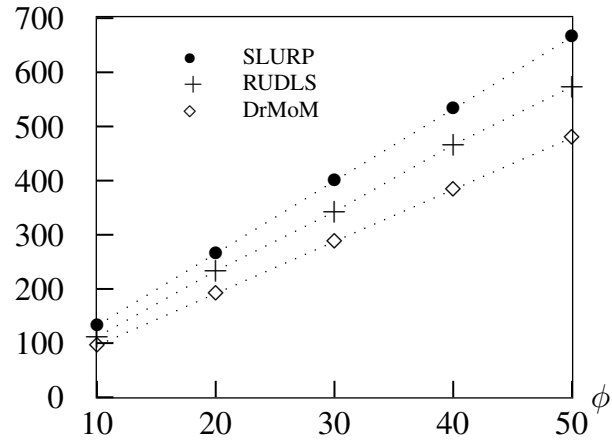

Fig. 6. Total communication cost vs. $\phi$ for DrMoM against SLURP and RUDLS.

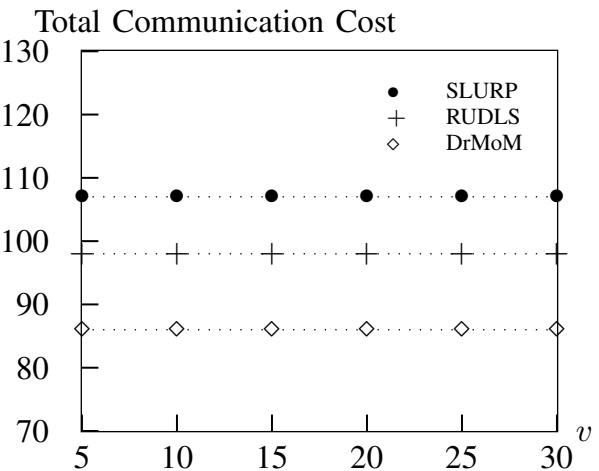

Fig. 7. Total communication cost vs. $v$ for DrMoM against SLURP and RUDLS.

communication cost incurred per time unit by DrMoM vs. SLURP and RUDLS as a function of the moving speed $v$ in the range of 5 to $30 \mathrm{~m} / \mathrm{s}$ for the scenario in which $n=100$ and $\phi=10$ packets $/ \mathrm{s}$. The communication cost is relatively insensitive to the moving speed $v$ because the data packet delivery cost $C_{d}$ dominates the location update $\operatorname{cost} C_{u}$ in the scenario considered. As can be seen in these two figures, DrMoM under the optimal setting (optimal $R_{l}$ and $R_{h}$ that together minimize the total communication cost) outperforms both SLURP and RUDLS over a wide range of moving speed and packet rate. This result clearly demonstrates the benefit of dynamically determining the optimal $R_{l}$ and $R_{h}$ for network cost minimization in DrMoM.

Fig. 8 compares the total communication cost incurred per time unit by DrMoM vs. SLURP and RUDLS as a function of the total number of mobile nodes $n$, or equivalently the node density, for the scenario in which $v=2 \mathrm{~m} / \mathrm{s}$ and $\phi=20$ packets $/ s$. As the figure illustrates, the total communication cost per time unit per user decreases as the node density increases because the success probability of local location queries increases as the number of neighbors increases. We again see that DrMoM is superior in terms of the total communication cost incurred per time unit per user. The advantage of DrMoM is particularly significant when the node density is relatively small. Again, the figure shows that the node density is a key parameter that affects the total communication cost incurred by a location management scheme for MANETs such as DrMoM, SLURP or RUDLS. 
Total Communication Cost

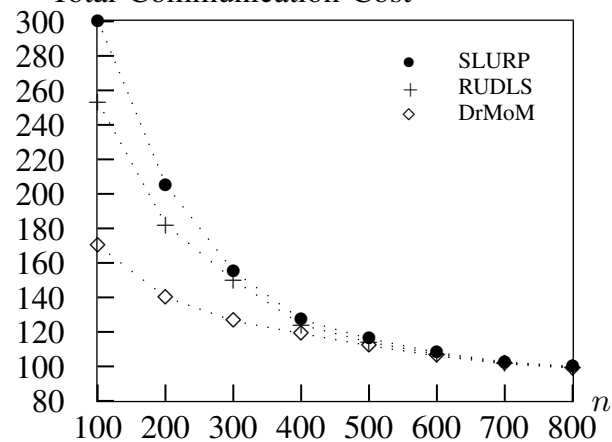

Fig. 8. Total communication cost vs. $n$ for DrMoM against SLURP and RUDLS.

\section{ApPlicABILITy AND CONCLUSION}

In this paper, we designed and analyzed a dual-region location management scheme (DrMoM) to provide efficient location service in MANETs. The novelty lies in dynamically identifying and applying the optimal home region size and local region size (defined by their respective radii denoted by $R_{h}$ and $R_{l}$ ) for each mobile node based on the mobile node's runtime mobility and service characteristics to minimize the overall network cost incurred for location management and data packet delivery. We developed a performance model to derive optimal $R_{h}$ and $R_{l}$ values as well as the total communication cost incurred by DrMoM. By means of a comparative performance study, we demonstrated that DrMoM outperforms existing location management schemes including SLURP and RUDLS.

The identification of optimal $R_{h}$ and $R_{l}$ settings to minimize the overall communication cost per user is performed at static time. One way to apply the results is to build a lookup table at static time listing the optimal $R_{h}$ and $R_{l}$ settings discovered over a perceivable range of parameter values characterizing a user's mobility and service behaviors. Then, at runtime, upon sensing mobility and service behavior changes matching with a set of parameter values, a mobile node can perform a simple table lookup operation augmented with extrapolation/interpolation techniques to determine and apply the optimal $R_{h}$ and $R_{l}$ settings to minimize the overall communication cost due to location management and packet delivery.

The performance model developed in thie paper is based on random movement. However, the analysis technique for identifying the optimal $R_{h}$ and $R_{l}$ settings is generally applicable. In the future, we plan to extend the analysis to consider other mobility models such as SWIM [24], utilizing more elaborated modeling techniques such as stochastic Petri nets $[25,26,27,28,29,30]$. This work also assumes that there are no malicious or selfish nodes performing attacks [31,32,33] to disrupt mobility management. We plan to investigate how trust management protocols such as $[34,35,36,37,38]$ can be used to select trustworthy nodes to serve as location servers to further enhance performance of dual-region location management in MANETs. Lastly we also plan to investigate how to further extend the design notion of integrated mobility and service management for cost minimization to other location-based services in MANETs such as cooperative data caching for mobile data access and mobile multicast with failure recovery $[39,40]$.

\section{ACKNOWLEDGEMENT}

This work is supported in part by the U. S. Army Research Office under contract number W911NF-12-1-0445.

\section{REFERENCES}

[1] Y. Li and I. R. Chen, "Design and performance analysis of mobility management schemes based on pointer forwarding for wireless mesh networks," IEEE Transactions on Mobile Computing, vol. 10, no. 3, pp. 349-361, 2011.

[2] Y. Li and I. R. Chen, "Mobility management in wireless mesh networks utilizing location routing and pointer forwarding," IEEE Transactions on Network and Service Management, vol. 9, no. 3, pp. 226-239, 2012.

[3] Y. Li and I. R. Chen, "Dynamic agent-based hierarchical multicast for wireless mesh networks," Ad Hoc Networks, vol. 11, no. 6, pp. 1683$1698,2013$.

[4] Y. Li and I. R. Chen, "Hierarchical agent-based secure and reliable multicast in wireless mesh networks," Computer Communications, vol. 36, no. 14, pp. 1515-1526, 2013.

[5] B. Gu and I. R. Chen, "Performance analysis of location-aware mobile service proxies for reducing network cost in personal communication systems," ACM Mobile Networks and Applications, vol. 10, no. 4, pp. 453-463, 2005.

[6] I. R. Chen, T. M. Chen, and C. Lee, "Performance evaluation of forwarding strategies for location management in mobile networks," The Computer Journal, vol. 41, no. 4, pp. 243-253, 1998.

[7] I. R. Chen, T. Chen, and C. Lee, "Agent-based forwarding strategies for reducing location management cost in mobile networks," ACM Mobile Networks and Applications, vol. 6, no. 2, pp. 105-115, 2001.

[8] I. R. Chen and D. C. Wang, "Regional Registration-Based mobile multicast service management in mobile IP networks," Wireless Personal Communications, vol. 54, pp. 635-649, Jun. 2009.

[9] H. Saleet, O. Basir, R. Langar, and R. Boutaba, "Region-based locationservice-management protocol for vanets," IEEE Transactions on Vehicular Technology, vol. 59, no. 2, pp. 917-931, 2010.

[10] M. Ayaida, H. Fouchal, L. Afilal, and Y. Ghamri-Doudane, "A comparison of reactive, grid and hierarchical location-based services for vanets," in IEEE Vehicular Technology Conference, Sept. 2012, pp. 1-5.

[11] E. Amar and S. Boumerdassi, "Enhancing location services with prediction," in IEEE Wireless Communications and Networking Conference, March 2011, pp. 593-598.

[12] C. Hsu and S. Wu, "An efficient cost based location service protocol for vehicular ad hoc networks," in IEEE international Conference on Communication, Networks and Satellite, July 2012, pp. 93-97.

[13] J. Zhou, J. Lu, and Y. Deng, "A grid-based predictive location service in mobile ad hoc networks," Future Wireless Networks and Information Systems, vol. LNEE 143, pp. 7-16, 2012.

[14] J. Zhou, J. Lu, S. Huang, and D. Luo, "An efficient region-based location service in mobile ad hoc networks," Journal of Information and Computational Science, vol. 9, no. 15, pp. 4531-4539, 2012.

[15] S. Das, H. Pucha, and Y. Hu, "Performance comparison of scalable location services for geographic ad hoc routing," in IEEE 24th Annual Joint Conference of the IEEE Computer and Communications Societies., Miami, FL, USA, 2005, pp. 1228-1239.

[16] S. M. Woo and S. Singh, "Scalable routing protocol for ad hoc networks," Wireless Networks, vol. 7, pp. 513-529, Sep. 2001.

[17] J. Li, J. Jannotti, D. S. J. D. Couto, D. R. Karger, and R. Morris, "A scalable location service for geographic ad hoc routing," in 6th ACM annual international conference on Mobile computing and networking, 2000, pp. 120-130.

[18] X. Wu, "VPDS: Virtual home region based distributed position service in mobile ad hoc networks," in 25th IEEE International Conference on Distributed Computing Systems, 2005, pp. 113-122.

[19] W. Wang and C. V. Ravishankar, "Hash-Based virtual hierarchies for scalable location service in mobile ad-hoc networks," Mobile Networks and Applications, vol. 14, pp. 625-637, Oct. 2009. 
[20] T. Park and K. G. Shin, "Optimal tradeoffs for location-based routing in large-scale ad hoc networks," IEEE/ACM Transactions on Networking, vol. 13, pp. 398-410, Apr. 2005.

[21] Z. Ye and A. A. Abouzeid, "Optimal stochastic location updates in mobile ad hoc networks," IEEE Transactions on Mobile Computing, vol. 10 , no. 5, pp. 638-652, 2011.

[22] S. Basagni, I. Chlamtac, V. R. Syrotiuk, and B. A. Woodward, "A distance routing effect algorithm for mobility (DREAM)," in ACM/IEEE international Conference on Mobile Computing and Networking, 1998, pp. $76-84$.

[23] L. E. Miller, "Connectivity properties of mesh and ring/mesh networks," in Wireless Communication Technologies, 2001.

[24] S. Kosta, A. Mei, and J. Stefa, "Small world in motion (SWIM): Modeling communities in ad-hoc mobile networking," in 7th IEEE Conference on Sensor, Mesh and Ad Hoc Communications and Networks, June 2010.

[25] C. Hirel, B. Tuffin, and K. S. Trivedi, "SPNP: Stochastic petri nets. version 6.0," in 11th International Conference on Computer Performance Evaluation: Modelling Techniques and Tools. London, UK: SpringerVerlag, 2000, pp. 354-357.

[26] I. R. Chen and D. C. Wang, "Analyzing Dynamic Voting using Petri Nets," in 15th IEEE Symposium on Reliable Distributed Systems, Niagara Falls, Canada, October 1996, pp. 44-53.

[27] I. R. Chen and D. C. Wang, "Analysis of replicated data with repair dependency," The Computer Journal, vol. 39, no. 9, pp. 767-779, 1996.

[28] S. T. Cheng, C. M. Chen, and I. R. Chen, "Dynamic quota-based admission control with sub-rating in multimedia servers," Multimedia Systems, vol. 8, no. 2, pp. 83-91, 2000.

[29] O. Yilmaz and I. R. Chen, "Utilizing call admission control for pricing optimization of multiple service classes in wireless cellular networks," Computer Communications, vol. 32, no. 2, pp. 317-323, 2009.

[30] I. R. Chen and T. H. Hsi, "Performance analysis of admission control algorithms based on reward optimization for real-time multimedia servers," Performance Evaluation, vol. 33, no. 2, pp. 89-112, 1998.

[31] R. Mitchell and I. R. Chen, "Effect of intrusion detection and response on reliability of cyber physical systems," IEEE Transactions on Reliability, vol. 62, no. 1, pp. 199-210, 2013.
[32] H. Al-Hamadi and I. R. Chen, "Redundancy management of multipath routing for intrusion tolerance in heterogeneous wireless sensor networks," IEEE Transactions on Network and Service Management, vol. 10, no. 2, pp. 189-203, 2013.

[33] I. R. Chen, A. P. Speer, and M. Eltoweissy, "Adaptive fault-tolerant QoS control algorithms for maximizing system lifetime of querybased wireless sensor networks," IEEE Transactions on Dependable and Secure Computing, vol. 8, no. 2, pp. 161-176, 2011.

[34] F. Bao, I. R. Chen, M. Chang, and J. H. Cho, "Hierarchical Trust Management for Wireless Sensor Networks and its Applications to TrustBased Routing and Intrusion Detection," IEEE Transactions on Network and Service Management, vol. 9, no. 2, pp. 169-183, June 2012.

[35] J. H. Cho, A. Swami, and I. R. Chen, "Modeling and analysis of trust management for cognitive mission-driven group communication systems in mobile ad hoc networks," in International Conference on Computational Science and Engineering, August 2009, pp. 641-650.

[36] J. H. Cho, A. Swami, and I. R. Chen, "Modeling and analysis of trust management with trust chain optimization in mobile ad hoc networks," Journal of Network and Computer Applications, vol. 35, no. 3, pp. 10011012, 2012.

[37] F. Bao, I. R. Chen, M. Chang, and J. H. Cho, "Trust-Based Intrusion Detection in Wireless Sensor Networks," in International Conference on Communications, Kyoto, Japan, June 2011, pp. 1-6.

[38] I. R. Chen, F. Bao, M. Chang, and J. H. Cho, "Trust Management for Encounter-based Routing in Delay Tolerant Networks," in Global Communications Conference, Miami, FL, USA, December 2010, pp. $1-6$.

[39] I. R. Chen, B. Gu, S. E. George, and S. T. Cheng, "On failure recoverability of client-server applications in mobile wireless environments," IEEE Transactions on Reliability, vol. 54, no. 1, pp. 115-122, 2005.

[40] S. E. George, I. R. Chen, and Y. Jin, "Movement-based checkpointing and logging for recovery in mobile computing systems," in 5th ACM International Workshop on Data Engineering for Wireless and Mobile Access, 2006, pp. 51-58. 